Esta obra forma parte del acervo de la Biblioteca Jurídica Virtual del Instituto de Investigaciones Jurídicas de la UNAM

\title{
VIEJOS Y NUEVOS RETOS DE LA DEMOCRACIA PARITARIA EN MÉXICO: LA PLENA IGUALDAD EN LA REPRESENTACIÓN POLÍTICA DE LOS GÉNEROS
}

Manuel GonzÁLEZ OROPEZA ${ }^{1}$

Carlos BÁEz SILvA ${ }^{2}$

El género femenino ha estado sometido a la violencia inherente a la discriminación en sus diversas formas, lo que le ha dificultado abrirse paso en la vida pública y en el campo de la participación política, pues en la lógica del poder éste contempla una compleja red de relaciones con intenciones claras y predefinidas para beneficiar al género masculino. Es por ello que la búsqueda de la equidad y paridad de género constituye un objetivo ético y de justicia social sobre la cual se erige la democracia, pero debe transformarse en un principio constitucional que rebase la igualdad formal para transformarse en un principio de acción positiva de gobierno. Hoy en día, hablar de género, de relaciones de género y de metas de equidad e igualdad de género, es hablar de derechos fundamentales que conciernen no sólo a las mujeres, sino también a los hombres. Se trata de alcanzar la plena igualdad entre las personas de ambos géneros.

Por supuesto que la participación de las mujeres en la política ayuda a fomentar la igualdad política de género. El nivel en el cual participa no importa tanto como su efectividad en la toma de decisiones públicas, lo cual ha quedado

\footnotetext{
${ }^{1}$ Magistrado de la Sala Superior del TEPJF; investigador nacional nivel III.

${ }^{2}$ Director del Centro de Capacitación Judicial Electoral del TEPJF; investigador nacional nivel I.
} 
Esta obra forma parte del acervo de la Biblioteca Jurídica Virtual del Instituto de Investigaciones Jurídicas de la UNAM www.juridicas.unam.mx

evidenciado en muchos casos, pues cuando las mujeres resultan electas para cargos públicos, aumenta la formulación de políticas que destacan la calidad de vida y reflejan las prioridades de las familias, las propias mujeres y las minorías étnicas y raciales. ${ }^{3}$ De igual manera es importante que los candidatos varones a ocupar cargos públicos sean capaces de tomar decisiones sensibles a la igualdad de género y que coadyuven con este ideal democrático y de justicia social.

De acuerdo con las estadísticas sobre la participación política de la mujer en América Latina, la proporción regional de mujeres diputadas está en un promedio del 20\%, las alcaldesas apenas llegan a un 5.3\%, mientras que en nuestro país, el porcentaje está por debajo del 6\%. Entre 1991 y 2008 doce países adoptaron leyes de cuotas para la participación política de las mujeres en las elecciones legislativas, cuyas cuotas varían entre 20 y 40\%. México ha establecido mandatos

${ }^{3}$ La literatura señala que las mujeres legisladoras tienden a comportarse diferente, cumpliendo con las expectativas: apoyan en mayor medida que sus contrapartes masculinos la legislación a favor de derechos reproductivos y cambio de roles de género (Norton, Noelle, "Uncovering the Dimensionality of Gender Voting in Congress", Legislative Studies Quarterly, núm. 24, vol. 1, 1999, pp. 65-86); apoyan programas de rehabilitación de criminales, mientras que los hombres suelen optar por castigos más severos (Kathlene, Lyn, "Alternative Views of Crime: Legislative Policymaking in Gendered Terms", Journal of Politics, núm. 57, vol. 3, 1995, pp. 696-723); dedican más tiempo al servicio de la comunidad que los hombres (Thomas, Sue, “The Effects of Race and Gender on Constituency Service", Western Political Quarterly, núm. 45, 1992, pp. 161-180); con mayor frecuencia toman en cuenta las opiniones de los ciudadanos y se preocupan en establecer vínculos con ellos (Fox, Richard L. y Schuhmann, Robert A., "Gender and Local Government: A Comparison of Women and Men City Managers", Public Administration Review, núm. 59, vol. 3, 1999, pp. 231-242); su estilo de gobierno enfatiza la búsqueda de coincidencias y cooperación (Tolleson Rinehart, Sue, “Do Women Leaders Make a Difference? Substance, Style and Perceptions", en Dodson, Debra (ed.), Gender and Policy Making, New Brunswick, N. J., Center for American Women and Politics, 1991), entre muchos otros (véase Lawless, Jennifer L. y Fox, Richard L., “Men Rule: The Continued Under-Representation of Women in U. S. Politics", Washington, D. C., Women \& Politics Institute, 2012, disponible en http://Www.american.edu/spa/wpi/upload/2012-Men-Rule-Report-web.pdf(consultado el 23 de julio de 2015)). Sin embargo, los estudios sobre los casos mexicanos señalan que las actividades a favor de género quedan subordinadas a los temas principales de la actividad política, como educación, salud, combate de la pobreza, etcétera (Rodríguez, Victoria E., Women's Participation in Mexican Political Life, Boulder, Colorado, Oxford, Westview Press, 1998 y Women in Contemporary Mexican Politics, Austin, University of Texas Press, 2003). Finalmente, se puede considerar que la expectativa de que las mujeres en el poder actúen de manera diferente y siempre a favor de los temas femeninos implica, en sí mismo, reproducción de un estereotipo y discriminación. 
Esta obra forma parte del acervo de la Biblioteca Jurídica Virtual del Instituto de Investigaciones Jurídicas de la UNAM

de ubicación como dispositivo que posibilita evitar que las mujeres candidatas sean colocadas en el fondo de las listas o en poblaciones y distritos electorales cuyo triunfo es poco probable (como miembros de un partido político).

Proyecciones recientes indican que de no aplicarse cuotas de género en los países de América Latina, habría que esperar hasta 2052 para que las mujeres ocuparan apenas el $40 \%$ de los puestos parlamentarios. Costa Rica y Ecuador son países que en 2011 habían llegado a la paridad en las candidaturas, mientras que nuestro país, con las reformas político-electorales de 2014, se aboca ya a consolidar la participación de las mujeres en la vida democrática del país, fundamentalmente desde los propios partidos políticos, quienes están obligados a garantizar que el $50 \%$ de sus candidaturas a legisladores federales y locales sean ocupados por mujeres. ${ }^{4}$

Previendo que pudieran hacerse "ajustes" que atentaran contra esta disposición, en ningún caso se admitirán criterios que tengan como resultado que

${ }^{4}$ Como los cambios en este sentido no eran suficientes, el entonces nuevo presidente de la República y los partidos políticos mayoritarios suscribieron el llamado "Pacto por México" en los primeros días de diciembre de 2012, con la finalidad de que en los primeros veinte meses de su administración, y durante el periodo de transición, se aprobaran estas transformaciones, las que fueron llamadas "reformas estructurales", entre ellas algunas en materia político-electoral; de entre todas ellas, una de las demandas sociales más urgentes para atender fue la de paridad de género en las candidaturas. Desde la reforma constitucional de octubre de 1953, en la cual la mujer pudo ejercer su derecho al sufragio, hasta ahora, sólo había conseguido desempeñar algunos cargos de elección popular; en otras ocasiones, fue utilizada para contender por una curul, que más tarde debió ceder a un compañero de partido, dando lugar al llamado caso de las "juanitas". Esta reforma busca cambiar desde sus cimientos la inequitativa situación entre hombres y mujeres, exigiendo una paridad absoluta y no simulada para contender por los cargos de elección popular en igualdad de condiciones y recursos. Para que las reglas de esta reforma político-electoral fueran consistentes con los cambios que debían operarse, fue necesario decretar primero la reforma constitucional de diversos artículos, la cual fue publicada en febrero de 2014, y meses más tarde, en mayo de ese mismo año, se hizo necesaria la expedición de la nueva Ley General en Materia de Delitos Electorales, la Ley General de Partidos Políticos, la Ley General de Instituciones y Procedimientos Electorales, y las modificaciones a la Ley General del Sistema de Medios de Impugnación en Materia Electoral, así como la Ley Orgánica del Poder Judicial de la Federación, y la Ley Federal de Responsabilidades Administrativas de los Servidores Públicos, además de que algunas instituciones del ámbito electoral también hicieron lo propio, como el INE que sustituyó al IFE, y el TEPJF que estableció la Sala Regional, entre otros cambios sustanciales. 
Esta obra forma parte del acervo de la Biblioteca Jurídica Virtual del Instituto de Investigaciones Jurídicas de la UNAM

a alguno de los géneros se le asignen de manera exclusiva aquellos distritos en los que el partido haya obtenido resultados bajos o que sean difíciles de remontar ante un adverso panorama. ${ }^{5}$ Es decir, varios países latinoamericanos ya están en la ruta constitucional de garantizar la participación igualitaria de hombres y mujeres en la contienda por los cargos de elección popular.

En general, la efectividad de una medida compensatoria y temporal como las cuotas no viene dada en forma mecánica; las cuotas aplicables a candidaturas en razón del género no se traducen en diputaciones en la misma proporción; existen otros factores que son determinantes para que ello ocurra. Entre algunos de los factores de mayor incidencia se pueden señalar:

1. El diseño electoral de cada entidad (equilibrio entre mayoría y representación proporcional en la conformación del congreso).

2. La existencia de un sistema de cuota y sanciones por incumplimiento explícitas.

3. Mecanismos de impugnación en materia de justicia electoral para mujeres y ciudadanía en lo general.

En la actualidad persiste una subrepresentación de las mujeres en los puestos de elección popular, cuyas cifras contrastan con el hecho de que las mujeres predominan dentro del total de la población del país. De acuerdo a los datos del Instituto Nacional Electoral, en 2014 las mujeres representaban el 52\% del total del padrón federal electoral (39714 251 hombres y 42760073 mujeres). ${ }^{6}$

A pesar de que es esperable que sea en el ámbito de los gobiernos municipales donde las mujeres tengan una participación ciudadana más activa, el número de

\footnotetext{
${ }^{5}$ Esta disposición sólo operaba para la elección de presidente de la República y diputados de mayoría relativa, ahora también es aplicable a los senadores por mayoría relativa; Capítulo III. De los cómputos distritales y de la declaración de validez de la elección de diputados de mayoría relativa, artículos 309 a 321 de la Ley General de Instituciones y Procedimientos Electorales, disponible en http://10.10.15.15/siscon/gateway.dII/nLegis/acion/nFederal//gipe?fn=documentframeset. $h$ tm $\$ f=$ templates $\$ 3.0$.

${ }^{6}$ Véanse las estadísticas de la lista nominal y del padrón electoral, disponibles en http://www.ine.mx/archivos3/portal/historico/contenido/Estadisticas_Lista_Nominal_y_Padron_Electoral/.
} 
Esta obra forma parte del acervo de la Biblioteca Jurídica Virtual del Instituto de Investigaciones Jurídicas de la UNAM

cargos de elección popular que ocupan es muy reducido. Para 2012 estadísticamente se reportaba que de los 2445 municipios que conforman la República mexicana, sólo el $7.16 \%$, es decir 175 eran gobernados por mujeres, y de los 1142 escaños totales de los congresos locales, únicamente 313 estaban ocupados por ellas. ${ }^{7}$

Por otra parte, en nuestro país sólo cuatro mujeres se han desempeñado como gobernadoras: Griselda Álvarez en Colima, en 1979; Beatriz Paredes en Tlaxcala, en 1987; Amalia García en Zacatecas, en 2004, e Ivonne Ortega Pacheco en Yucatán, en 2009. Rosario Robles en el Distrito Federal, en 1997, y Dulce María Sauri en Yucatán, en 1992, tuvieron el carácter de jefa de Gobierno y gobernadora interinas, respectivamente. Para septiembre de este año, el Estado de Sonora contará con la única mujer que estará al frente de un Poder Ejecutivo en toda la República, Claudia Artemiza Pavlovich Arellano, para el periodo 20152021.8

En el ámbito internacional, México ha suscrito diversos compromisos que prescriben un mayor acceso y participación en el ejercicio del poder público a la mujer, siendo las más importantes:

1. La Convención de los Derechos Políticos de la Mujer, ratificada por México el 23 de marzo de 1981.

2. La Convención sobre la Eliminación de todas las Formas de Discriminación contra las Mujeres (CEDAW-ONU). México ratificó esta Convención el 23 de marzo de 1981, año en el que entró en vigor en nuestro país.

3. El Pacto Internacional de Derechos Civiles y Políticos, ratificado por México el 23 de marzo de 1981.

\footnotetext{
${ }^{7}$ Observatorio de Participación Política de las Mujeres en México, 2015, disponible en http://aplicaciones.inmujeres.gob.mx/observatorio/.

${ }^{8}$ El jefe de Gobierno de la Ciudad de México, Miguel Ángel Mancera, en los recientes cambios a su gabinete, también designó a una mujer como secretaria de Gobierno de la ciudad, Dora Patricia Mercado Castro, quien años atrás había contendido como candidata presidencial por el Partido Alternativa Socialdemócrata en el 2006.
} 
Esta obra forma parte del acervo de la Biblioteca Jurídica Virtual del Instituto de Investigaciones Jurídicas de la UNAM

4. El Convenio 169 de la OIT sobre Pueblos Indígenas y Tribales en Países Independientes, ratificado por México el 13 de agosto de 1990.

5. La Convención Americana sobre Derechos Humanos (Pacto de San José, Costa Rica), ratificada por México el 2 de marzo de 1981.

6. Convención Interamericana sobre Concesión de los Derechos Políticos a la Mujer, ratificada por México el 24 de marzo de 1981.

La Convención Belem Do Pará, ratificada por México el 19 de junio de 1998, ha reconocido que la violencia contra la mujer viola la dignidad humana y lleva necesariamente a la discriminación por género, y ha establecido que la mujer tiene derecho a una vida sin violencia, al reconocimiento y al ejercicio de sus derechos humanos. Al efecto, deben darse patrones socioculturales de conducta de respeto a la mujer, fomentar programas educativos para erradicar prejuicios y discriminación, e impulsar programas de capacitación para la administración de la justicia.

Además de estos instrumentos internacionales, la Conferencia Mundial sobre la Mujer que se celebró en Beijing en 1995, de la que surgió la Declaración y el llamado Programa de Acción de Beijing, firmado por la Unión Europea y adoptado en la IV Conferencia Mundial de las Naciones Unidas sobre las Mujeres, celebrada el 7 de septiembre de 1995, en los que se afirma que: "el concepto de democracia no adquirirá un sentido real y dinámico hasta que las orientaciones políticas y las legislaciones nacionales no sean definidas en común por los hombres y las mujeres", considerándose el "poder y la toma de decisiones" uno de los doce campos críticos de intervención, estableciéndose las medidas a poner en práctica, incluso acciones positivas, por los organismos internacionales y nacionales para asegurar una igual representación de hombres y mujeres.

La armonización de la legislación nacional conforme a los tratados y convenciones internacionales en favor de los derechos humanos de las mujeres, comprende no sólo la emisión de leyes, sino también la modificación de reglamentos, códigos civiles, penales, administrativos y de procedimientos, que 
Esta obra forma parte del acervo de la Biblioteca Jurídica Virtual del Instituto de Investigaciones Jurídicas de la UNAM

garanticen el acceso de las mujeres a la justicia en condiciones de igualdad. En agosto de 2015, 28 estados de la República mexicana habían incorporado el Principio de Igualdad entre Mujeres y Hombres en sus Constituciones, y 30 estados el Principio de no Discriminación. ${ }^{9}$

La Constitución Política de los Estados Unidos Mexicanos establece desde 1991, en su artículo 4o., la igualdad ante la ley de hombres y mujeres, y desde 2001, en sus artículos 10. y 20., la prohibición de toda discriminación por género y el reconocimiento a los derechos de los pueblos indígenas, entre ellos el decidir sus formas internas de organización, la aplicación de sus propios sistemas normativos, elegir a sus propias autoridades y representantes, y por supuesto, acceder de manera plena a la jurisdicción del Estado. ${ }^{10}$ La reforma constitucional de 2014 incorporó al artículo 41 el principio de paridad de género, imponiendo a los partidos políticos la obligación de garantizar la integración paritaria de las listas de candidatos a legisladores federales y locales (artículo 41, base I de la CPEUM). Recientemente, en mayo de 2015, una nueva reforma modificó el contenido del artículo 2o. constitucional, señalando que los pueblos y las comunidades indígenas tienen derecho de aplicar sus propios sistemas normativos en la regulación y solución de sus conflictos internos, "sujetándose a los principios generales de esta Constitución, respetando las garantías individuales, los derechos humanos $\mathrm{y}$, de manera relevante, la dignidad e integridad de las mujeres" (artículo 20., apartado A, base II de la CPEUM).

\footnotetext{
${ }^{9}$ Véanse los textos de las constituciones estatales a partir del 11 de agosto de 2015.

${ }^{10}$ Elegir, de acuerdo con sus normas, procedimientos y prácticas tradicionales, a las autoridades 0 representantes para el ejercicio de sus formas propias de gobierno interno, garantizando que las mujeres y los hombres indígenas disfrutarán y ejercerán su derecho de votar y ser votados en condiciones de igualdad, así como a acceder y desempeñar los cargos públicos y de elección popular para los que hayan sido electos o designados, en un marco que respete el pacto federal y la soberanía de los estados. En ningún caso las prácticas comunitarias podrán limitar los derechos político-electorales de los ciudadanos en la elección de sus autoridades municipales. Véase
}

http://10.10.15.15/siscon/gateway.dIl/nLegis/acion/nFederal/constitucion?fn=documentframeset.htm\$f=templates\$3.0. Última reforma al artículo 20., inciso III, del 22 de mayo de 2015. 
Esta obra forma parte del acervo de la Biblioteca Jurídica Virtual del Instituto de Investigaciones Jurídicas de la UNAM

También la Convención sobre la Eliminación de todas las Formas de Discriminación contra la Mujer, en su artículo 7o., establece la obligación de los Estados de tomar las medidas apropiadas para eliminar la discriminación; en particular, para garantizar la igualdad de condiciones con los hombres respecto al derecho a votar en las elecciones y ser elegibles para los órganos públicos de elección popular, ocupando los cargos públicos en todos los planos gubernamentales.

Estos preceptos constitucionales en materia electoral se expresan en el establecimiento en la legislación general de paridad de géneros, en el registro de candidaturas e integración de los órganos de dirección de los partidos políticos. La Ley General de Partidos Políticos (LGPP) señala que éstos deben asegurar la participación efectiva de ambos géneros, tanto en la integración de sus órganos como en la postulación de candidatos. Para ello, cada partido determinará los criterios para garantizar la paridad en las candidaturas, en los que deberá tomar en cuenta la expectativa de ganar la elección (artículos 33.3, 4 y 5, LGPP). Las listas de candidatos a los cargos de elección popular, para la integración del Congreso de la Unión, los congresos de los Estados y la Asamblea Legislativa del Distrito Federal, deberán ser integradas por el 50\% de candidatas mujeres y el $50 \%$ de hombres (artículo 232.3, LEGIPE). Además, las fórmulas de candidatos por mayoría relativa y por representación popular deben ser integradas por personas del mismo género (artículo 14.4, LEGIPE). Finalmente, la LGPP establece que ningún partido podrá postular una fórmula —integrada por un mismo géneroen los distritos dónde han obtenido la votación más baja (LGPP, artículo 3.5). ${ }^{11}$

En nuestro país, las mujeres cuentan con instrumentos legales para la defensa

\footnotetext{
${ }^{11}$ En apoyo de este propósito, la LGPP también estipula que los partidos políticos habrán de destinar al menos 3\% del financiamiento público ordinario que reciben a través del Instituto Nacional Electoral, a la capacitación, promoción y desarrollo del liderazgo político de las mujeres. México ha avanzado en el campo legislativo en la cuestión de capacitación de mujeres, determinando que una parte de los recursos públicos asignados al sostenimiento institucional y permanente de los partidos políticos debe orientarse a la capacitación de líderes femeninas y a la promoción de la participación activa de la mujer en la política, ya sea en las ciudades y en el campo.
} 
Esta obra forma parte del acervo de la Biblioteca Jurídica Virtual del Instituto de Investigaciones Jurídicas de la UNAM

de su derecho a participar de manera directa en el ejercicio del poder público. El juicio para la protección de los derechos político-electorales del ciudadano, como instrumento legal, permite a los ciudadanos impugnar todos aquellos actos de los partidos políticos y de las autoridades electorales que violenten sus derechos, entre otros casos, en su vertiente de ser votadas a puestos de elección popular y para ejercer cargos en los órganos electorales. Con relación a los partidos políticos, tenemos que están formados de diversos elementos y cumplen múltiples funciones, tales como representar a la sociedad y propulsar el sistema político, en su doble papel de agrupar las élites y elegirlas, y aplicar los derechos políticos de los ciudadanos. Por todo ello, los partidos son un referente obligado en el reconocimiento de los derechos políticos de la mujer.

Es importante señalar que esos cambios constitucionales y legislativos derivan, en una medida importante, del esfuerzo del Tribunal Electoral del Poder Judicial de la Federación por fortalecer la aplicación y efectividad de la cuota de género. Una de las decisiones que son directamente vinculadas con la reforma 2014 se dio durante el proceso electoral 2011-2012.

Al inicio del proceso electoral, en octubre de 2011, el Consejo General del IFE emitió el Acuerdo CG327/2011, mediante el cual se establecen los criterios aplicables para el registro de candidaturas para el proceso federal electoral 20112012. El acuerdo establecía, entre otras, una excepción a la aplicación de las cuotas, para el caso de las candidaturas de mayoría relativa que sean resultado de un proceso de elección democrático (conforme al artículo 219.2 del Cofipe, que estaba vigente en aquel momento). Además, incluía una recomendación, señalando que los partidos deben procurar que la fórmula completa se integre por candidatos del mismo género.

Inconformes con la regulación aprobada por el IFE, un grupo de mujeres presentaron un juicio ciudadano, solicitando al TEPJF modificación del acuerdo con la finalidad de fortalecer la implementación de las cuotas. Las impugnantes combatían la excepción a la aplicación a la cuota y solicitaban que ésta se volviera una obligación firme, y no una sugerencia a los partidos políticos en cuanto a la 
Esta obra forma parte del acervo de la Biblioteca Jurídica Virtual del Instituto de Investigaciones Jurídicas de la UNAM

integración de las fórmulas por candidatos de un solo género.

En la sentencia referida, SUP-JDC-12624/2011, la Sala Superior del TEPJF emitió criterios que permitieron un importante avance de la cuota de género. En primer lugar, la Sala declaró la inconstitucionalidad de la regla incluida en el artículo 219.2 del Cofipe, que señalaba que los partidos podían incumplir con la cuota cuando sus candidatos fuesen electos mediante un procedimiento democrático, conforme a sus estatutos. El acuerdo del IFE definía como procedimiento democrático todo aquél en el que la elección de las candidaturas se realizara de forma directa por la militancia del partido o por la ciudadanía, o de forma indirecta a través de una convención o asamblea de delegados electos ex profeso por dicha militancia. La Sala sostuvo que el Instituto no puede, en el ejercicio de la facultad reglamentaria, modificar o alterar el contenido de una ley, es decir, los reglamentos no pueden contener mayores supuestos, ni crear nuevas limitantes a las previstas en la ley. Además, al asumir que los procedimientos para la designación de candidatos previstos en los estatutos vigentes de los partidos políticos son democráticos (en cumplimiento con las obligaciones establecidas por la legislación y jurisprudencia), en todo caso y sin importar el procedimiento específico, los partidos tienen la obligación de cumplir con la cuota de género en todas las postulaciones de mayoría relativa.

Finalmente, la Sala Superior estableció que, dentro del $40 \%$ de las candidaturas correspondientes al género minoritario, la fórmula completa (propietario y suplente) tendría que ser del mismo género. ${ }^{12}$ Esta decisión permitió acabar con el fenómeno de las llamadas "juanitas": mujeres postuladas por los partidos políticos que, al momento de ser electas, renunciaban a favor de los candidatos suplentes, quienes eran hombres. Esa medida, al garantizar que, si llegaran a presentarse vacantes en los propietarios, éstos serían sustituidos por personas del mismo género, permite conservar el equilibrio de género no sólo en

\footnotetext{
${ }^{12}$ Más tarde, ese criterio fue incorporado en la Jurisprudencia 16/2012: CUOTA DE GÉNERO. LAS FÓRMULAS DE CANDIDATOS A DIPUTADOS Y SENADORES POR AMBOS PRINCIPIOS DEBEN INTEGRARSE CON PERSONAS DEL MISMO GÉNERO.
} 
Esta obra forma parte del acervo de la Biblioteca Jurídica Virtual del Instituto de Investigaciones Jurídicas de la UNAM

las candidaturas, sino también en la ocupación de los cargos.

La sentencia, al eliminar la excepción a la cuota y obligar a presentar fórmulas de candidatos del mismo género (en al menos $40 \%$ de las postulaciones), dio un paso clave en la implementación de las cuotas. En la elección de 2012 se logró el $37 \%$ de representación femenina en la Cámara de Diputados, por primera vez superando el umbral mínimo necesario para que la presencia de un grupo en un cuerpo colegiado alcance los niveles necesarios para conseguir influencia en la toma de decisiones, que los estudios ubican en el 30\%. En menos de veinte años, a partir de las modificaciones legislativas y actuación de las autoridades, el porcentaje de representación de las mujeres en el Congreso pasó del 15\% inicial hasta el 37\% en la integración 2012-2015.

Las nuevas reglas de paridad fueron implementadas, por primera vez, durante el proceso electoral 2014-2015, y han sido objeto de múltiples pronunciamientos por parte del TEPJF. En principio, podemos destacar dos ámbitos en los que se destacan los esfuerzos del Tribunal a favor de la equidad de género: aplicación del principio de paridad a nivel municipal y en la integración de los órganos legislativos.

En ese sentido, es importante señalar que ni la Constitución, ni la legislación general incluyen la aplicación de la paridad de género para las postulaciones en el ámbito municipal. Las legislaciones locales de algunas entidades federativas prevén las medidas afirmativas para las elecciones municipales, sin embargo, no todos y no siempre de la misma manera. Algunos estados han introducido obligaciones fuertes, incluyendo la paridad horizontal y vertical (Campeche), mientras que otros introdujeron sólo una modalidad (Chiapas-vertical).

Sin embargo, durante el proceso electoral, la Sala Superior ha establecido que el principio de paridad de género debe aplicarse a la integración de listas de candidatos tanto vía mayoría relativa (horizontal) como de representación proporcional (vertical). La Sala Superior, que sostuvo que es obligación de las autoridades electorales dotar de efectividad al principio de paridad de género, para que sea aplicado en la postulación de las candidaturas a las presidencias 
Esta obra forma parte del acervo de la Biblioteca Jurídica Virtual del Instituto de Investigaciones Jurídicas de la UNAM

municipales (SUP-REC-46/2015). A partir de esa sentencia, en los demás casos relativos a las postulaciones a los cargos de presidente municipal, las salas del TEPJF han estado implementando ese criterio, exigiendo que las candidaturas estén lo más cercanas a la paridad de género posible. En cuanto a la paridad en las listas de representación proporcional a nivel municipal, el TEPJF ha sostenido que también es obligatorio en todos los casos, aun cuando la legislación local no lo prevé expresamente, ya que el no hacerlo significaría incumplimiento con el mandato constitucional: "desconocer la alternancia entre todos los integrantes de la planilla sin exclusión, como medida óptima para lograr el efectivo acceso de ambos géneros al poder municipal en condiciones igualitarias, conduce a desconocer el principio de paridad de género" (SDF-JRC-17/2015).

El criterio general adoptado por la Sala Superior señala que "la paridad horizontal y vertical a nivel municipal es un mandato constitucional y convencional y, por tanto, su aplicación resulta incuestionable. Es decir, es de observancia obligatoria" y que su finalidad es "el adecuado equilibrio en la participación política de hombres y mujeres, en el caso, en la integración de los Ayuntamientos y con ello lograr la participación política efectiva en la toma de decisiones del mencionado ente público colegiado, en un plano de igualdad sustancial, con el objetivo de consolidar dicha paridad de género como práctica política" (SUP-REC-128/2015).

Finalmente, es muy importante destacar el caso de registro de candidatos en la elección en Chiapas, donde sólo un partido (Morena) cumplió con el principio de paridad. Para obligar a los demás actores a su cumplimiento, la Sala Superior del TEPJF obligó a los partidos a realizar las sustituciones necesarias dentro de un plazo de 48, y suspendiendo las campañas hasta que se completen las correcciones de las listas (SUP-REC-294/2015).

En cuanto a la paridad en la integración de los órganos legislativos, el primer caso que la establece tiene que ver con la elección de diputados locales en Coahuila en 2014. Al revisar el caso y percatarse de un desequilibrio entre los hombres y las mujeres en la asignación final de escaños, la Sala Monterrey 
Esta obra forma parte del acervo de la Biblioteca Jurídica Virtual del Instituto de Investigaciones Jurídicas de la UNAM

determinó que la integración total del congreso debe ser paritaria y que, para lograrlo, es viable recorrer el orden de candidatos en las listas de RP, privilegiando a las candidaturas femeninas. La Sala Monterrey argumentó que la postulación paritaria de candidatos para la integración del Congreso debe traducirse en un mecanismo que permita a las mujeres acceder de forma efectiva a los cargos públicos, incluso a pesar del orden que establezcan los partidos políticos en sus listas de representación proporcional, sin que esto signifique una violación a la autodeterminación de los partidos. Sostuvo también que con ello:

No se priva de un derecho adquirido al candidato que ocupe el primer lugar de la lista, pues con independencia de la posición que tenga en el listado, su derecho a detentar un escaño estará limitado en la medida que su nombramiento como diputado impida que en la integración del Congreso del Estado, se respete el principio de igualdad y así como las acciones afirmativas en materia de equidad de género.

Además de que "la acción afirmativa consistente en la conformación paritaria y alternada de las listas de postulación a diputados por el principio de representación proporcional, tiene como finalidad, constituir un mecanismo efectivo para garantizar el ejercicio de las mujeres a su derecho humano de acceso a los cargos de elección popular" (SM-JRC-14/2014).

La Sala Superior, en la sentencia SUP-REC-936/2014, confirmó el criterio de la Sala Monterrey, sosteniendo que los principios de paridad de género, igualdad sustantiva y no discriminación, constituyen el fundamento para sostener que la cuota prevista para la postulación de candidaturas debe trascender a la asignación de diputaciones por el principio de representación proporcional y a la integración total del Congreso. Sin embargo, en la misma sentencia modificó el desarrollo del procedimiento realizado por la Sala Monterrey, armonizando la equidad de género con el derecho de autodeterminación de los partidos políticos, de acuerdo a lo siguiente:

- La autoridad deberá tener presente si es par o impar el número de curules por repartir, así como las diputaciones alcanzadas por las mujeres a través del principio de mayoría, pues estos elementos le sirven de sustento para determinar el número de diputaciones por el principio de representación 
Esta obra forma parte del acervo de la Biblioteca Jurídica Virtual del Instituto de Investigaciones Jurídicas de la UNAM

proporcional que corresponderán a las mujeres, a fin de alcanzar la paridad de género en la integración del Poder Legislativo. Eso significa que en la integración de los congresos de número impar de diputados, es aceptable que el número de diputados hombres sea ligeramente superior al número de diputadas mujeres, acercándose lo más posible a la paridad.

- Cuando sea necesario modificar el orden de prelación propuesto por los partidos, se debe empezar por el partido que habiendo registrado un hombre en primer lugar de la lista obtuvo el menor porcentaje de votación, dado que en la asignación de curules por el principio de representación proporcional, el porcentaje de votación constituye uno de los elementos principales para determinar el derecho de los partidos a obtener una curul por ese principio, lo que es congruente con garantizar en la mayor medida la autoorganización de los partidos, a través del respeto del orden de prelación de la lista.

De esta manera, la Sala Superior señaló que debe realizarse un paso adicional en la asignación de escaños por RP, en el cual la autoridad debe verificar si el resultado obtenido en la elección de diputados de mayoría relativa es cercano a la paridad, y en cualquier caso, realizar la asignación de escaños de RP a manera de ajuste, pretendiendo lograr un resultado lo más cercano a la paridad posible, ya en la integración total del legislativo.

Como podemos observar, las Salas del TEPJF se han tomado una postura firme a favor de la paridad de género, obligando a los partidos políticos a cumplir con ese principio en la integración de las listas de candidatos a los cargos de elección popular, con especial énfasis sobre las postulaciones en el ámbito municipal. A partir de la reforma y la actuación firme de las autoridades electorales se espera que, este año, la integración de las autoridades legislativas y municipales, por primera vez en la historia, quede muy cercana a la paridad entre los géneros.

\section{FUENTES CONSULTADAS}

Fox, Richard L. y SCHUHmanN, Robert A., “Gender and Local Government: A Comparison of Women and Men City Managers", Public Administration Review, 
Esta obra forma parte del acervo de la Biblioteca Jurídica Virtual del Instituto de Investigaciones Jurídicas de la UNAM

núm. 59, vol. 3, 1999.

GonzÁlez OropezA, Manuel, “Los partidos políticos como promotores de la participación de género", Revista de Derecho Estasiológico. Ideología y Militancia, México, UNAM, Facultad de Estudios Superiores AragónCoordinación del Posgrado en Derecho, núm. 1, enero-junio de 2013.

KATHLENE, Lyn, "Alternative Views of Crime: Legislative Policymaking in Gendered Terms", Journal of Politics, núm. 57, vol. 3, 1995.

LAWLESS, Jennifer L. y Fox, Richard L., “Men Rule: The Continued Under-

Representation of Women in U. S. Politics", Washington, D. C., Women \& Politics Institute, 2012, disponible en http://www.american.edu/spa/wpi/upload/2012-Men-Rule-Report-web.pdf (consultado el 23 de julio de 2015).

NoRTON, Noelle, "Uncovering the Dimensionality of Gender Voting in Congress", Legislative Studies Quarterly, núm. 24, vol. 1, 1999.

RodríGUez, Victoria E., Women's Participation in Mexican Political Life, Boulder, Colorado, Oxford, Westview Press, 1998.

_-_, Women in Contemporary Mexican Politics, Austin, University of Texas Press, 2003.

ThomAs, Sue, "The Effects of Race and Gender on Constituency Service", Western Political Quarterly, núm. 45, 1992.

Tolleson Rinehart, Sue, “Do Women Leaders Make a Difference? Substance, Style and Perceptions", en Dodson, Debra (ed.), Gender and Policy Making, New Brunswick, N. J., Center for American Women and Politics, 1991. 